\title{
Facing sampling techniques as an optimal design problem
}

\section{Christos P. Kitsos}

\begin{abstract}
Department of Informatics and Computer Engineering, University of West Attica, Agiou Spiridonos 28, 12243, Aigaleo, xkitsos@uniwa.gr
\end{abstract}

\section{SUMMARY}

The aim of this paper is to investigate and discuss the common points shared, in their line of development, by both Sampling Theory and Design of Experiments. In fact, Sampling Theory adopts the main optimality criterion of the Optimal Design of Experiments, the minimization of variance, i.e. Doptimality. There is also an approach based on c-optimality, as far as ratio estimates are concerned, in Design of Experiments, and the A-optimality involved in a proposed Sampling technique. It is pointed out that the L2 norm is mainly applied as a distance measure.

Key words: sampling Theory, alphabetic optimality criteria, ratio estimates, mean square error

\section{Introduction}

In principle, statistics has its vital roots in probability theory at one end, while at the other end it branches into applied problems involving collecting and analyzing various data, from almost all scientific fields. At this point statistics becomes embedded into sampling techniques. Sampling offers an early chance to distinguish statistics from mathematical analysis, as it offers plans, designs, errors, randomness and inference. Errors are the core value of statistics, as well as the main distribution; it is assumed that they follow the normal distribution. At early stages it was applied to astronomy, to geodesy, and later to social sciences and economics.

The sense of "population" was discussed early by A. Quetelet for social problems, and only when Maxwell and Boltzman adopted the idea into mathematical physics was it also used by Galton and Pearson in genetics and biometrics respectively. As late as 1866, pioneering work on sweet peas offered the first explicit indeterministic example. 
Experiment and sample design were founded at almost the same time, with the early work of Fisher (1934) and Neyman and Pearson (1928). In this paper we shall investigate the fact that both Optimum Experimental Design Theory and Sampling Techniques mainly follow a common approach: "to minimize the variance" or a function of the variance. There are different estimators of the variance (section 3), with nice properties. In practice only one well-known estimator is adopted.

A heretical approach was published by Papadakis (1937), who somehow, in his early work, adjusted (corrected) the response $y_{i}$, for a given treatment, taking into account the values of the "neighbourhood area" of the treatment in an agricultural experiment. Bartlett $(1938,1978)$ considered this approach, which is independent of the usual normal assumption and based on no independent observations and no randomization; see for details Kounias (2016).

In this paper we discuss the appropriate background of an experimental design under the randomization approach of Fisher and the classical sampling techniques, as developed by Cochran, in section 2. In section 3 , we investigate the common points of these different statistical approaches, while in section 4 ratio estimates are tackled.

\section{Background}

Let $\mathrm{U} \subseteq \mathrm{R}^{k}$ be the set in which the predictor (input) variables take their values, known as a design space. Let $\Xi$ be a family of measures $\xi$ such that $\xi(u) \geqslant 0$ and $\int \xi d u=1$. We shall refer to $\xi$ as a design measure and the pair $(U, \xi)$ as a design space (Silvey 1980), provided that we are working in a continuous framework.

For the discrete case the framework is not that different.

Let us consider an exact design with $n$ points of support and $\tau_{i}$ the (integer) number of trials in experimental conditions $x_{i}, i=1,2, \ldots, n$ with $N$ being the total number of trials. Then the design can be written as:

$$
\xi_{n}=\left\{\begin{array}{llll}
x_{1} & x_{2} & \ldots & x_{n} \\
\tau_{1} \frac{1}{N} & \tau_{2} \frac{1}{N} & \ldots & \tau_{n} \frac{1}{N}
\end{array}\right\}
$$

with $\sum \tau_{i}=N$ and let $p_{i}=\frac{1}{N} \tau_{i}, i=1,2, \ldots, n$, the proportion of observations concerning the point $x_{i}$. 
The design measure $\xi_{n}$ specifies the experimental conditions as well as the "proportion" of the experimental effort devoted to each condition. The optimum design may be:

$$
\xi_{k}^{*}=\left\{\begin{array}{cccc}
x_{1}^{*} & x_{2}^{*} & \ldots & x_{k}^{*} \\
p_{1}^{*} & p_{2}^{*} & \ldots & p_{k}
\end{array}\right\}, \quad \sum_{l=1}^{k} p_{l}^{*}=1
$$

Example For the linear regression model for the third-degree polynomial

$$
y=\beta_{0}+\beta_{1} x+\beta_{2} x^{2}+\beta_{3} x^{3}, \quad x \in U=[-1,1]
$$

with $\beta=\left(\beta_{0}, \beta_{1}, \beta_{2}, \beta_{3}\right) \in B \subseteq \mathrm{R}^{4}, B$ the parameter space. The optimum design is:

$$
\xi_{4}^{*}=\left\{\begin{array}{rrrr}
-1.00 & -0.447 & 0.447 & 1.00 \\
0.25 & 0.250 & 0.250 & 0.25
\end{array}\right\}
$$

(see Federov 1972).

Let us denote by $\operatorname{Cov}(E)$ the covariance matrix estimated for considering the parameter $\theta$ under experiment $E$. Recall that we say that an experiment $E_{1}$ is preferred to an experiment $E_{2}$, in notation $E_{1}>E_{2}$, if the difference $\operatorname{Cov}\left(E_{2}\right)-\operatorname{Cov}\left(E_{1}\right)$ is a positive definite matrix or if:

$$
\left|\operatorname{Cov}\left(E_{1}\right)\right|<\left|\operatorname{Cov}\left(E_{2}\right)\right|
$$

(see Federov 1972). There are optimality criteria based on different functions of $\operatorname{Cov}(E)$ known as alphabetic optimality criteria (see Federov 1972 for the linear case, Kitsos 1986 for the non-linear case), but we mainly focus on D-optimality. Recall that the dispersion matrix of the best linear unbiased estimate offers a determinant which is the smallest among all the determinants of the dispersion matrix, for all linear unbiased estimation.

Let us consider the stratified sampling where the total population (universe) is divided, under the imposed criteria, into $L$ strata, the $l$-th stratum contains a total number $N_{l}$ of units, and let the sample units at the $l$-th stratum be $l=1,2, \ldots, L$ so that:

$$
N=\sum_{l=1}^{L} N_{l}, \quad n=\sum_{l=1}^{L} n_{l}, \quad f_{l}=\frac{n_{l}}{N_{l}} .
$$

Let the measurement be $y_{l i}, l=1,2, \ldots, L, i=1, \ldots, N_{l}$, while the weight of stratum $l$ in the sample $n$ is $w_{l}=n_{l} / n$. 
Cochran (2011), Deming (1990), Kish (1965), Som (1973), among others, define the main characteristics of different sampling techniques. Briefly, these techniques are:

SS1: Proportional (Bowley) allocation, with:

$$
\begin{aligned}
& n_{l}=\frac{n}{N} N_{l} \\
& \operatorname{Var}\left(\bar{y}_{\text {prop }}\right)=\frac{1-f}{n} \sum_{l=1}^{L} w_{l} s_{l}^{2}, \quad f=\frac{n}{N}
\end{aligned}
$$

with $n$ being the sample size.

When $s_{l}^{2}=s_{\text {within }}^{2}:=s_{w}^{2}$

$$
\operatorname{Var}\left(\bar{y}_{\text {prop }}\right)=\frac{s_{w}^{2}}{n}(1-f)
$$

SS2: Neyman Allocation, with:

$$
\begin{aligned}
& n_{l}=n \frac{w_{l} s_{l}}{\sum_{l} x_{l} s_{l}} \\
& \operatorname{Var}\left(\bar{y}_{\text {Neyman }}\right)=\frac{1}{N}\left(\sum_{l=1}^{L} w_{l} s_{l}\right)^{2}-\frac{1}{N} \sum_{l=1}^{L} w_{l} s_{l}^{2}
\end{aligned}
$$

SS3: A cost can be involved, offering a constraint to the optimization problem.

Let $\bar{y}_{\text {srs }}$ be the estimator obtained from a simple random sampling with variance $\operatorname{Var}\left(\bar{y}_{\text {srs }}\right)$. In principle the simple random sample (SRS) is a subset of the population with $n$ observations (known as sample size), where the individuals of the subset have been chosen once, randomly, with the same probability. The SRS is the basic technique among a number of sampling techniques (Cochran 2011, Som 1973). Then it can be proved that:

$$
\operatorname{Var}\left(\bar{y}_{\text {Neyman }}\right) \leqslant \operatorname{Var}\left(\bar{y}_{\text {prop }}\right) \leqslant \operatorname{Var}\left(\bar{y}_{\text {srs }}\right)
$$

Due to this relation (5), recalling (3) and (1) and the definition of SRS, the following holds. 
Proposition 1. Let $E_{N e y}, E_{p r}, E_{s r s}$ be an experiment design with the Neyman allocation, the proportional allocation for the stratified sampling experiments, and $E_{\text {srs }}$ the simple random sampling experiment. Then

$$
E_{N e y}<E_{p r}<E_{\text {srs }}
$$

with the corresponding design measures:

$$
\begin{gathered}
\xi_{N e y}=\frac{n_{l}}{n}=\frac{w_{l} s_{l}}{\sum_{l} w_{l} s_{l}} \\
\xi_{p r}=\frac{n_{l}}{n}=\frac{N_{L}}{N} \\
\xi_{\text {srs }}=\frac{1}{n}
\end{gathered}
$$

Moreover, as concerns the systematic sampling, let the estimated response be $\bar{y}_{s y}$ with variance $\operatorname{Var}\left(\bar{y}_{s y}\right)$. The order between $\operatorname{Var}\left(\bar{y}_{s y}\right)$ and $\operatorname{Var}\left(\bar{y}_{\text {srs }}\right)$ is highly dependent on whether the sample in systematic sampling is heterogeneous or homogeneous. In the former situation:

$$
\operatorname{Var}\left(\bar{y}_{s y}\right) \leqslant \operatorname{Var}\left(\bar{y}_{s r s}\right)
$$

and in the latter the dual. Therefore the following holds:

Proposition 2. If the sampling units are heterogeneous (HS) in a systematic sampling, then the corresponding experiment $E_{s y}$ is preferable to the SRS experiment, i.e.,

$$
\begin{aligned}
E_{s y}>E_{\text {srs }}, \quad E_{s y} \text { is hs } \\
\text { with } \quad \xi_{s y}=\xi_{s r c}=1 / n .
\end{aligned}
$$

As the criterion under consideration is the variance, for one parameter, optimality refers to minimum variance, i.e., to D-optimality (Silvey 1980).

But still with only one parameter under consideration, all of the alphabetic optimality criteria (D-, A-, G-) coincide.

In Sampling Theory (Cochran 2011, Som 1973, among others), most of the imposed optimality criteria are results of minimizing the variance of the parameter of interest. The cost function in sampling acts as a constraint for the optimalization problem. The constrained optimization problem, with emphasis on optimum design theory, has been extensively discussed by Torsney (1981), while the non-linear design problem can be considered as a constrained one (Kitsos 1986). A typical non-linear problem is the ratio problem, discussed in the next section. 


\section{Sampling techniques}

There are different sampling techniques, but all are based on the same principle: survey sampling tries to reflect the presentation of the population by randomization of the sample over the entire defined population.

Pseudo-experiments are those designs which are neither completely randomized nor have realistic treatments. Moreover, for the demarcation of the different strata, different rules of thumb are imposed - so as to "restrict the design area". The main approaches close to pseudo-experiments are:

(i) Dalentius-Curney rule: Require via the construction for the strata the rule: $N_{l} S_{l}=N_{l} \sigma_{l}$ in simple random sampling with replacement.

(ii) Ekman rule: Reduce the lack of information requiring the equalization of $N_{l} R_{l}$, with $R_{l}$ being the range of the response in the $l$-stratum.

(iii) Mahalanobis-Hansen-Hurwitz-Malow rule: Form the strata to make $\bar{Y}_{l}$ the same.

All of the above rules have an experimental feature, and it is not that easy to form the data in such a way.

Moreover the design of experiments reflects the randomization of treatments over the subjects in the sample. It is usual in practice to consider the "pros and cons" and to make a choice between sample surveys sacrificing randomization of treatment or experiments sacrificing representation. The smoking problem, as well as the lung cancer one, can be tackled in different studies, while some (not only nuclear) experiments can never be representative.

Let us consider a given sample $x_{1}, x_{2}, \ldots, x_{n}$ of size $n$, with $\bar{x}$ being the average of $x_{i}, i=1,2, \ldots, n$, coming from a normal population, with mean $\mu$ and variance $\sigma^{2}$. Let us define the distance of each observation $x_{i}$ from the average $\bar{x}$, say $d_{i}^{k}$ with $d_{i}^{k}=\left|x_{i}-\bar{x}\right|^{k}$ and $d^{k}=\sum_{i=1}^{n}\left|x_{i}-\bar{x}\right|^{k}$, while the distance from the true mean is $D_{i}^{k}=\left|x_{i}-\mu\right|^{k}, D^{k}=\sum_{i=1}^{n}\left|x_{i}-\mu\right|^{k}$, $k=1,2$. With $k=1$ we refer to the $L_{1}$ norm, and for $k=2$ to the $L_{2}$ norm.

Then the estimators, well-known in practice, of the variance $\sigma^{2}$ are:

$$
s^{2}=\frac{1}{n-1} d^{2}, \quad s_{*}^{2}=\frac{1}{n} d^{2}
$$

and

$$
M A D=\frac{1}{n} d^{1}, \quad M A D_{*}=\frac{1}{n} \sqrt{\frac{\pi}{2}} d^{1}
$$


with $M A D$ being the known Mean Absolute Deviation. An unbiased estimator of $\sigma$ is the statistic $S_{0}$ :

$$
S_{0}=\frac{\sqrt{n} \Gamma\left(\frac{n}{2}\right)}{\sqrt{2} \Gamma\left(\frac{n+1}{2}\right)} s
$$

with $E\left(S_{0}\right)=\sigma$.

Moreover for the sample $x_{1}, x_{2}, \ldots, x_{n}$ coming from the population $\operatorname{Pop}\left(\mu, \sigma^{2}\right)$, an unbiased estimator of $s^{2}$ may be $S_{c}^{2}(x)=c \sum_{i=1}^{n-1}\left(x_{i+1}-x_{i}\right)^{2}$ with $c=\frac{1}{2(n-1)}$.

Indeed, as:

$$
\sigma^{2}=E\left[S_{c}^{2}(x)\right]=c E\left\{\sum_{i=1}^{n-1}\left(x_{i+1}-x_{i}\right)^{2}\right\}=c(n-1) 2 \sigma^{2} .
$$

If the observations are independent and identically $N\left(\mu, \sigma^{2}\right)$ distributed, based on the fact that:

$$
E\left(D_{i}^{1}\right)=\sigma \sqrt{\frac{2}{\pi}}, \quad i=1,2, \ldots, n
$$

the statistic:

$$
T_{c}(x)=\frac{1}{n} \sqrt{\frac{\pi}{2}} D^{1}
$$

also provides an unbiased estimator of $\sigma^{2}$. Moreover the following holds.

Proposition 3. For the unbiased estimator of $\sigma^{2}$ as in (13) the variance is:

$$
\operatorname{Var}\left(T_{c}(x)\right)=\frac{\sigma^{2}}{2 n}(\pi-2)=\operatorname{Var}(\tilde{X})-\operatorname{Var}(\bar{X})
$$

with $\tilde{X}$ being the median.

Indeed:

$$
\operatorname{Var}\left(T_{c}(x)\right)=\frac{\pi}{2 n^{2}} \operatorname{Var}\left(x_{i}-\mu\right)=\frac{\pi}{2 n^{2}} n \frac{\sigma^{2}}{\pi}(\pi-2)=\frac{\sigma^{2}}{2 n}(\pi-2)
$$

Corollary 1. If the data follow a symmetric distribution, then $\operatorname{Var}\left(T_{c}(x)\right)=0$. 
The various forms of the estimated variance can be useful, as the variance is what needs to be minimized. Eventually the $s^{2}$ is applied in both sampling and design theory. The $L_{2}$ norm introduced by Legendre in 1805 is commonly adopted. The $L_{1}$ norm introduced by Boscovich in 1757 is not much used in practice (Dodge 1987), while the $L_{\infty}$ norm introduced by Laplace in 1799 is not applied in either sampling or design (see also Wynn 1977a, 1977b). Based on a minimum distance principle, Blyth (1970) proved that all of the main estimation methods follow this approach, under the $L_{2}$ norm.

\section{Ratio estimates}

An essential application of a ratio estimator was considered by Laplace in 1786, to estimate the population of France. Ratio estimates were first used in 1662 in England by the founder of demography, John Graunt.

In principle, the ratio estimate of the random variables $X$ and $Y$ is most effective when $Y$ is regressed on $X$ and the regression line passes through the origin, so as to give $Y=R X$. As usual the population size is $N$ and the sample size $n$; for the ratio $R$ the estimate is:

$$
\hat{R}=\frac{y}{x}=\frac{\bar{y}}{\bar{x}} .
$$

Moreover, the following can be obtained:

$$
\hat{\bar{Y}}=\frac{y}{x} \bar{X}, \quad \hat{Y}=\frac{y}{x} X
$$

and for $n$ large enough it holds that $E(\hat{R}) \simeq R$ and

$$
\operatorname{Var}(\hat{R})=\frac{(1-f)}{n(n-1) \bar{X}^{2}} \sum_{i=1}^{n}\left(y_{i}-R x_{i}\right)^{2}:=g(R), \quad f=\frac{n}{N}
$$

That is, the variance of the estimate $\hat{R}$ depends on the parameter required to be estimated, namely $R$. This is the case in all non-linear Design of Experiments (DoE) problems (Kitsos 1986). Other approaches have also been considered (Siscodia and Dwivedi 1981, Reddy 1974). We can overcome this difficulty, as in DoE with two-stage design (Kitsos 1986, 2002).

Let the covariance of $X$ and $Y$ be:

$$
\rho=\operatorname{Cov}(X, Y)
$$


Then, for the standard deviations of $X$ and $Y, s_{X}$ and $s_{Y}$ respectively, and for $R$ and $\rho$, the following holds:

$$
\rho=R \frac{s_{X}}{s_{Y}} \quad \text { then } \quad V\left(\bar{y}_{R}\right)<V\left(\bar{y}_{L R}\right)
$$

with $\bar{y}_{L R}$ being the Linear Regression estimate and $\bar{y}_{R}$ the Ratio estimate.

However, when:

$$
\left(\rho s_{Y}-R s_{X}\right)^{2}>0 \text { then } V\left(\bar{y}_{L R}\right)<V\left(\bar{y}_{R}\right)
$$

The above relation implies that both $y_{L R}$ and $\bar{y}_{R}$ can lead to a Doptimal design depending on the relation between $\rho$ and $R$, then extending Som (1973) for relative designs, the following holds.

Proposition 4. Consider the ratio estimate problem as above and let

$$
w=\rho s_{y}-R s_{x}=\left|\begin{array}{cc}
\rho & R \\
s_{x} & s_{y}
\end{array}\right|
$$

Then:

$$
w^{2}=\left\{\begin{aligned}
0 & \text { ratio design preferable, } E_{R}<E_{L R} \\
>0 & \text { linear regression design optimal, } E_{L R}<E_{R}
\end{aligned}\right.
$$

The linear regression optimal design may be a preferable one, with minimum variance, but it is not a unique one, as the ratio design can be a "better one" (in terms of minimum variance).

The calibration problem has been extensively discussed by Kitsos (1999), and has even been considered as a robust linear problem (Kitsos and Müller 1995). We in fact refer to the calibration problem as a ratio estimate, and it is briefly discussed below.

Consider the regression model:

$$
\eta=E(y \mid x)=\beta_{0}+\beta_{1} x, \quad x \in U=[-1,1]
$$

with $U$ being the (transformed) design space.

The aim is to estimate the value of $x=x_{0}$, given that the response is known, $k$ say, i.e. $\eta=k$ such that: 


$$
x_{0}=\frac{k-\beta_{0}}{\beta_{1}}
$$

To obtain the estimate $\hat{x}_{0}$ of $x_{0}$, use the criterion: $\min \operatorname{Var}\left(\hat{x}_{0}\right)$.

This can be obtained either through a D-optimal design or adopting c-optimal design, Kitsos (1986).

For the D-optimal design, which is actually a one-stage design, the variance can be evaluated approximately (Kitsos 2002) as:

$$
V_{D}=\frac{1}{n \beta_{1}^{2}}\left(x_{0}^{2}+1\right)
$$

while for the c-optimal design the approximate variance is:

$$
V_{C}=\left\{\begin{array}{cl}
\frac{1}{n \beta_{1}^{2}}, & \left|x_{0}\right|<1 \\
\frac{1}{n \beta_{1}^{2}} x_{0}^{2}, & \text { otherwise }
\end{array}\right.
$$

with $n$ being the number of observations.

Evaluating the efficiency $\frac{V_{C}}{V_{D}}$, one can see that it is $50 \%$ at the end points, and $100 \%$ at the center (Kitsos 1995).

The main difference comparing ratio estimates in Sampling and Calibration in Regression is that we move from the discrete case to the continuous case, where c-optimality is easier to apply. Hence ratio estimates in Sampling Theory (see also Reddy 1974) are mainly associated with D-optimality, rather than c-optimality as can be obtained in a calibration problem.

\section{Ratio estimates}

Time has passed since the first census made with the aid of computers (Beale 1962), or interest in coefficients of variation (Searl 1964), but still the Mean Square Error (MSE) (Tarter 1986) remains valuable in both Sampling and Experiments.

Optimal experimental designs, either linear (Federov 1972, Silvey 1980, among others), or non-linear, especially concerning the ratio (Kitsos 1986, 1999, Tin 1965, Valliant 1985, Dash 2015), have been extensively discussed.

There are also a number of contributions concerning the realization of sampling techniques as an optimal design (Wynn 1977a, 1977b, Kish 1976, 
Cheng and Li 1987), without specifying precisely the similarities, as has been attempted in this paper.

In Sampling Theory, the optimality criteria are based on minimizing the well-known variance (Cochran 2011, Deming 1990, Som 1973, Kendall and Stuart 1976), despite the fact that there are a number of proposed variances.

This is a result of the extensively used distance methods (Blyth 1970) based on Euclidean distance, rather than the $L_{1}$-Norm (Dodge 1987). The cost function in sampling (Cochran 2011) acts as a constraint for the general optimalization problem (Torsney 1981).

Therefore we tackled the sampling problem as a design problem, with the ratio estimate to be a non-linear problem like the calibration problem: the minimum variance approach is adopted in both cases, and a two-stage design has been introduced and adopted in both cases. That is, in principle, both schools of thought follow the minimum variance approach. The candidate sampling pattern provides the model to form an experiment which we need, usually, to optimize it.

As a simple case that conforms to both approaches, consider the systematic sampling in two dimensions, which, adjusted into a square, leads to the Latin square experimental design.

Moreover the variance in both non-linear situations - sampling ratio estimate and experiment theory - depends on the unknown parameter we wish to estimate. This creates a problem. Either an estimator is needed, or a two-stage approach can be adopted to re-estimate the initial guess.

The optimal design measures, as well as the variances, for different sampling techniques have been discussed in this paper in the light of Doptimality.

C-optimality has not been adopted in sampling, although two-stage designs are widely used. As far as A-optimality is concerned, an A-optimal strategy is the optimal strategy under the superpopulation model (Cheng and Li 1987), while the strategy introduced by Rao, Hartley and Cochran (1962) is not A-optimal.

Both Cox (1952) and Atkinson (1969) proved that working with Papadakis' (1937) different sampling scheme, treatments can be obtained providing A-optimal estimators. Moreover; Kunert and Martin (1987) proved that this design is also D-optimal. G-optimality can be considered through the equivalence theorem with $\mathrm{D}$, while for a one-parameter estimator the alphabetic designs coincide.

Therefore, in this paper optimal design characteristics have been pro- 
vided that fit appropriately to the sampling approach, sharing both common goals, but certainly with different sizes of observations under study.

\section{Acknowledgements}

I would like to thank Dr. C.S. Nisiotis, University of West Attica, for useful discussions during the preparation of this paper.

\section{REFERENCES}

Atkinson A.C. (1969): The use of residuals as a concomitant variable. Biometrika $56,33--41$.

Bartlett M.S. (1938): The approximate recovery of information from replicated field experiments with large blocks. The Journal of Agricultural Science 28, $418-427$.

Bartlett M.S. (1978): Nearest neighbour models in the analysis of field experiments. Journal of the Royal Statistical Society: Series B (Methodological) 40, 147158.

Beale, E. M. L. (1962): Some uses of computers in operational research. Industrielle Organisation $31,51-52$.

Blyth R.C. (1970): On the Inference and Decision Models of Statistics. The Annals of Mathematical Statistics 41(3), 1034-1058.

Cheng C.-S., Li K.-C. (1987). Optimality criteria in survey sampling. Biometrika $74,337-345$.

Cochran W.G. (2011): Sampling techniques; John Wiley \& Sons.

Cox D. (1952): Some recent work on systematic experimental designs. Journal of the Royal Statistical Society: Series B 14, 211-219.

Dash P. (2015): Comparison of Some Almost Unbiased Ratio Estimators. Int. Res. J. of Eng. and Tech. (IRJET) 2, 767--772.

Deming W.E.(1990): Sample design in business research; John Wiley \& Sons.

Dodge Y.(1987): Statistical data analysis based on the L1-norm and related methods; North-Holland, New York.

Fedorov V.V. (1972): Theory of optimal experiments; Academic Press, London.

Fisher R. (1934): Randomisation, and an old enigma of card play. The Mathematical Gazette 18, 294-297.

Kendall M G., Stuart A. (1976): The advanced theory of statistics. Hafner Pub. Co. New York.

Kish L. (1965): Survey sampling. John Wiley: New York.

Kish L. (1976): Optima and proxima in linear sample designs. Journal of the Royal Statistical Society: Series A (General) 139, 80 - 95.

Kitsos C.P. (2002): The simple linear calibration problem as an optimal experimental design. Communications in Statistics-Theory and Methods 31, 11671177.

Kitsos C.P. (1999): Calibration. Encyclopedia of Statistical Science, by Kotz, Samuel, Read, Campbell B and Banks, D 3, 62--65. 
Kitsos C.P., Müller C.H. (1995): Robust linear calibration. Statistics 27, 93-106.

Kitsos C.P. (1986): Design and Inference in Nonlinear Problems. Unpublished PhD Thesis. University of Glasgow.

Kounias, S. (2016): Jean Papadakis (1903-1997). Proceedings of the 29th Panhellenic Stat. Conference, Thessaloniki and Naousa, 4-7 May 2016, 103-107.

Kunert J., Martin R.J. (1987): On the optimality of finite Williams II(a) designs. The Annals of Statistics 15, 1604--1628.

Neyman J., Pearson E.S. (1928): On the use and interpretation of certain test criteria for purposes of statistical inference. Biometrika 20, 263-294.

Neyman J. (1934): On the two different aspects of the representative method: the method of stratified sampling and the method of purposive selection. J. R. Stat. Soc. 97, 558-625.

Papadakis J.S. (1937): Méthode statistique pour des expériences sur champ. Institute d' Ameliration Plantes a Salonique. (Greece). Bulletin Scientifique 23, $13-29$.

Rao J.N.K., Hartley H.O., Cochran W.G. (1962): A simple procedure of unequal probability sampling without replacement. Journal of the Royal Statistical Society: Series B (Methodological) 24, 482--491.

Reddy V.N. (1974): On a transformed ratio method of estimation. Sankhya C 36, $59-70$.

Searl D.T. (1964): The utilization of a known coefficient of variation in the estimation procedure. Journal of the American Statistical Association 59, 12251226.

Silvey S. (1980): Optimal design. Chapman and Hall.

Siscodia B., Dwivedi V. (1981): Modified ratio estimator using coefficient of variation of auxiliary variable. Journal-Indian Society of Agricultural Statistics, $13-18$.

Som R.K. (1973): A manual of sampling techniques. Heinemann, London.

Tarter M.E. (1986): Mean integrated squared error sampling. Journal of the American Statistical Association 81, 234--242.

Tin M. (1965): Comparison of some ratio estimators. Journal of the American Statistical Association 60, 294-307.

Torsney B. (1981): Algorithms for a constrained optimization problem with applications in statistics and optimum design., Ph.D. Thesis, University of Glasgow.

Valliant R. (1985): Nonlinear prediction theory and the estimation of proportions in a finite population. Journal of the American Statistical Association 80, 631-641.

Wynn H.P. (1977a): Minimax purposive survey sampling design. Journal of the American Statistical Association 72, 655-657.

Wynn H.P., In Statistical Decision Theory and Related Topics; by Gupta, S. and Moore, D. (Eds). Academic Press. New York. Elsevier: 1977b, 471—478. 